\title{
First-Order Light Deflection by Einstein-Strauss Vacuole Method
}

\author{
Debasish Saha, ${ }^{1}$ Amarjit Tamang, ${ }^{1}$ Ramil Izmailov, ${ }^{2}$ Carlo Cattani, ${ }^{3}$ and Kamal K. Nandi ${ }^{1,2}$ \\ ${ }^{1}$ Department of Mathematics, University of North Bengal, Siliguri 734 013, India \\ ${ }^{2}$ Ya.B. Zel'dovich International Center for Astrophysics, BSPU, Ufa 450000, Russia \\ ${ }^{3}$ Department of Mathematics, University of Salerno, Via Ponte Don Melillo, 84084 Fisciano, Italy
}

Correspondence should be addressed to Kamal K. Nandi; kamalnandi1952@yahoo.co.in

Received 20 February 2013; Accepted 15 July 2013

Academic Editor: Jose Antonio De Freitas Pacheco

Copyright (C) 2013 Debasish Saha et al. This is an open access article distributed under the Creative Commons Attribution License, which permits unrestricted use, distribution, and reproduction in any medium, provided the original work is properly cited.

We resolve here an outstanding problem plaguing conformal gravity in its role in making consistent astrophysical predictions. Though its static spherically symmetric solution incorporates all the successes of Schwarzschild gravity, the fit to observed galactic rotation curves requires $\gamma>0$, while the observed increase in the Schwarzschild light deflection by galaxies appears to demand $\gamma<0$. Here we show that, contrary to common knowledge, there is an increase in the Schwarzschild deflection angle in the vicinity of galaxies due purely to the effect of $\gamma>0$, when the idea of the Einstein-Strauss vacuole model is employed. With the inconsistency now out of the way, conformal gravity should be regarded as a good theory explaining light deflection by galaxies.

\section{Introduction}

The metric exterior to a static spherically symmetric distribution in Weyl conformal gravity has been obtained by Mannheim and Kazanas [1]. Recently, the solution has been used to fit rotation curves of many galaxy samples [2] as well as to predict the maximal size of galaxies [3]. The metric, which we call Mannheim-Kazanas-de Sitter (MKdS) metric, reads $(G=c=1)$

$$
\begin{gathered}
d \tau^{2}=-B(r) d t^{2}+\frac{1}{B(r)} d r^{2}+r^{2}\left(d \theta^{2}+\sin ^{2} \theta d \varphi^{2}\right), \\
B(r)=1-\frac{2 M}{r}+\gamma r-k r^{2},
\end{gathered}
$$

where $M$ is the central mass and $k$ and $\gamma$ are arbitrary constants that could be appropriately fixed by using the fit to rotation curves. For distances neither too small nor too large, the above mentioned metric is a good approximation. Now, there could be three possible ways to calculate light deflection in the above spacetime. First, the conventional calculations for light deflection show that the constant $k$ does not appear in the relevant equations, leading finally to the two way deflection as [4]

$$
2 \epsilon=\frac{4 M}{r_{0}}-\gamma r_{0}
$$

where $r_{0}$ is the distance of closest approach. The difficulty is that the fit to observed rotation curve requires $\gamma>0$, and for consistency all other astrophysical observations should respect this sign. Now, the observed light deflection by a galaxy is always more than the Schwarzschild value $4 M / r_{0}$, and hence to avoid the negative contribution in (3), one must demand $\gamma<0$ [4]. Thus there appears an inconsistency from the usual method. The second option is to use the RindlerIshak method [5], which is based on the realization that conventional methods do not apply to asymptotically nonflat spacetimes as the limit $r \rightarrow \infty$ makes no sense in it. Their original method of invariant angle is most appropriate in such situations, but it has an as yet unnoticed difficulty on the galactic scales, as explained below. Ishak et al. [6] thereafter improved the calculations using the Einstein-Strauss vacuole model and this provides us with the third and best option in our opinion. The purpose of this paper is to use the vacuole model to show that there is an increase in the Schwarzschild 
deflection angle in the vicinity of galaxies due purely to the effect of $\gamma>0$.

\section{Rindler-Ishak Original Method}

The bending angle in general is defined by $\epsilon=\psi-\varphi$. Rindler and Ishak considered the case $\varphi=0$ so that the deflection angle is $\epsilon=\psi$ given by

$$
\tan \psi=\frac{r \sqrt{B(r)}}{|A|},
$$

where $A(r, \varphi)=d r / d \varphi$. With $u=1 / r$, the photon trajectory from (2) is given by

$$
\frac{d^{2} u}{d \varphi^{2}}=-u+3 M u^{2}-\frac{\gamma}{2} .
$$

As evident, $k$ has disappeared from the above equation. Solving perturbatively to first order in $M$, we have

$$
\begin{gathered}
u=\frac{1}{r}=\frac{\sin \varphi}{R}-\frac{\gamma}{2}+\frac{M}{4 R^{2}}\left[6+3 R^{2} \gamma^{2}-3 R \gamma(\pi-2 \varphi) \cos \varphi\right. \\
+2 \cos 2 \varphi-6 R \gamma \sin \varphi],
\end{gathered}
$$

where the parameter $R$ is related to the closest approach distance $r_{0}$ at $\varphi=\pi / 2$. Note that the usual Schwarzschild orbit equation $u=(1 / R) \sin \varphi+\left(M / 2 R^{2}\right)(3+\cos 2 \varphi)$ is obtained at $\gamma=0$. From (6), we can find $r$ at $\varphi=0$ as

$$
r=\frac{4 R^{2}}{X}
$$

where

$$
X \equiv 8 M-3 \pi R M \gamma-2 R^{2} \gamma+3 M R^{2} \gamma^{2} .
$$

Also, at $\varphi=0$, we find that

$$
|A|=\frac{16 R^{3}}{X^{2}} .
$$

It can be seen again that, at $\gamma=0$, we recover the Schwarzschild values $r=R^{2} / 2 M$ and $|A|=R^{3} / 4 M^{2}$. Using the value of $r$ from (7) and $|A|$ from (9), we get from (4) the required one way deflection angle for small $\psi$ in the first order in $\gamma$ as

$$
\begin{aligned}
\psi \simeq \frac{r \sqrt{B(r)}}{|A|} & =\frac{2 M}{R} \sqrt{Y}+f(M, R, \sqrt{Y}) \gamma, \\
Y & \equiv 1-\frac{4 M^{2}}{R^{2}}-\frac{k R^{4}}{4 M^{2}} .
\end{aligned}
$$

For the solar system, $M_{\odot}=1.48 \times 10^{5} \mathrm{~cm}, R_{\odot}=6.96 \times$ $10^{10} \mathrm{~cm}$, and taking $k=9.54 \times 10^{-54} \mathrm{~cm}^{-2}$ [2], we get $k R^{4} / 4 M^{2}=1.15 \times 10^{-22}$, which is far less than unity allowing a series expansion of $\sqrt{Y}$. The expansion up to correct secondorder contribution also follows. One also obtains a positive contribution to deflection due to the Mannheim value of $\gamma$ $\left(=3.06 \times 10^{-30} \mathrm{~cm}\right)$, namely, $+13 M^{2} \gamma / R \simeq 1.25 \times 10^{-29}$, a very insignificant effect. So there is no difficulty in the local scale; the difficulty arises when one goes to the galactic scale. Typically, say for UGC0128, the disc plus bulge plus hydrogen gas mass $M=5.16 \times 10^{15} \mathrm{~cm}$, and the scale length $R_{0}=$ $2.07 \times 10^{22} \mathrm{~cm}[2]$, we have $k R_{0}^{4} / 4 M^{2}=7.40$, which makes $\psi$ imaginary and the expansion terms from $\sqrt{Y}$ unreliable! Thus the method needed improvement and that is exactly what was done by Ishak et al. [6] in their subsequent implementation of the Einstein-Strauss vacuole. We shall follow this below.

\section{Einstein-Strauss Vacuole: Improved Rindler-Ishak Method}

The suggestion of a cut-off boundary was first made by Edery and Paranjape [4]. We shall assume that the spacetime (2) (or MKdS vacuole) is exactly embedded in a FLRW universe, and the matching between the two spacetimes occurs at a radius $r_{b}$, the boundary of the vacuole. We shall further assume that light deflection occurs only inside the MKdS vacuole and that once the light transitions are out of the vacuole and into FLRW universe, light ceases to bend. The junction conditions are prescribed by Einstein and Strauss [7] as

$$
\begin{gathered}
r_{b} \text { in MKdS }=a(t) r_{b} \text { in FLRW, } \\
M_{\mathrm{MKdS}}=\frac{4 \pi}{3} r_{b}^{3} \text { in MKdS } \times \rho_{\text {matter in FLRW }} .
\end{gathered}
$$

Note that conformal gravity was used to fit galactic rotation curves without assuming any hypothetical dark matter, and the global positive constants $\gamma$ and $k$ can be assigned suitable numerical values to obtain the required fit [2]. Therefore, by $M_{\text {MKdS }}$, we mean only the luminous mass contributed by the bulge, disc, and hydrogen gas inside the vacuole. In general, the vacuole radius $r_{b}$ would also change due to cosmic expansion, but we shall consider $r_{b}$ at that particular instant $t_{0}$ of cosmic epoch when the light ray happens to pass the point of the closest approach $r_{0}$ to the lens. Current observations suggest that the universe is spatially flat so that we can take $\rho_{\text {matter in FLRW }}=\rho_{\text {critical }}=3 H_{0}^{2} / 8 \pi=$ $1.1 \times 10^{-29}\left(H_{0} / 75 \mathrm{~km} / \mathrm{sec} / \mathrm{Mpc}\right)^{2} \mathrm{gm} / \mathrm{cm}^{3}$. The bending is not sensitive to the exact value of $\rho$. Normalizing the scale factor to $a\left(t_{0}\right)=1$ and dropping suffixes, the above prescription translates to

$$
r_{b}=\left(\frac{3 M}{4 \pi \rho}\right)^{1 / 3}
$$

where $M$ is the luminous lens mass expressed in units of sun's mass $M_{\odot}=1.989 \times 10^{33} \mathrm{gm}$. Clearly, for any given lens, $r_{b}$ is much larger than the closest approach distance $r_{0} \simeq R$, often expressed in terms of impact parameter.

Assume a small entry angle $\varphi_{b}$ radian at the vacuole boundary radius $r_{b}$ such that $\sin \varphi_{b} \simeq \varphi_{b}$ and $\cos \varphi_{b} \simeq 1$. Then, (6) gives

$$
\frac{1}{r_{b}}=-\frac{\gamma}{2}+\frac{\varphi_{b}}{R}+M\left[\frac{2}{R^{2}}-\frac{3 \pi \gamma}{R}+\frac{3 \gamma^{2}}{4}\right] .
$$


Similarly,

$$
A_{b}\left(r_{b}, \varphi_{b}\right)=\frac{r_{b}^{2}}{R}-\frac{2 M \varphi_{b} r_{b}^{2}}{R^{2}} .
$$

The small one way bending angle $\psi$ becomes

$$
\psi=\frac{r_{b} \sqrt{B\left(r_{b}\right)}}{\left|A_{b}\right|}-\varphi_{b},
$$

which expands in first order in $\gamma$ as

$$
\begin{aligned}
\psi & =-\varphi_{b}+\left(\frac{2 M+R \varphi_{b}}{R-2 M \varphi_{b}}\right) \sqrt{Z}+g\left(M, R, \varphi_{b}, \sqrt{Z}\right) \gamma \\
& \equiv \psi_{0}+g \gamma,
\end{aligned}
$$

where

$$
\begin{gathered}
Z \equiv 1-\frac{4 M^{2}}{R^{2}}-\frac{2 M \varphi_{b}}{R}-\frac{k R^{4}}{\left(2 M+R \varphi_{b}\right)^{2}}, \\
\psi_{0} \equiv-\varphi_{b}+\left(\frac{2 M+R \varphi_{b}}{R-2 M \varphi_{b}}\right) \sqrt{Z}
\end{gathered}
$$

Given any $M$ in grams of a galaxy or cluster lens, one can easily calculate its $r_{b}$ using (12). Then using the value of scale length $R$ of the lens, and putting the value of $r_{b}$ in the equation (rewritten from (13))

$$
\varphi_{b}=R\left[\frac{1}{r_{b}}+\frac{\gamma}{2}-M\left(\frac{2}{R^{2}}-\frac{3 \pi \gamma}{4 R}\right)\right],
$$

it can be verified that $\varphi_{b}$ is indeed very small justifying its small approximation at the vacuole boundary entry. Furthermore, the term involving $k$ in (17) is also much smaller than unity thus allowing for a legitimate expansion of $Z$. The difficulty involving the $k$ dependent term mentioned in Section 2 now disappears, as the numerical calculations with typical galactic values show. We also note that for any galaxy lens the contribution due to $g \gamma$ is negative but extremely small and is of no interest. Expanding the remaining term $\psi_{0}$ in (18), we get

$$
\psi_{0} \simeq \frac{2 M}{R}+M\left(\frac{\varphi_{b}^{2}}{R}+\frac{k R}{\varphi_{b}^{2}}-k R\right)-\frac{k R^{2}}{2 \varphi_{b}} .
$$

With $\varphi_{b} r_{b} \simeq R$, the last term can be recognized as the term $-\Lambda R r_{b} / 6$ obtained in [6] contributing negatively. The middle term coupled to mass $M$ and $\gamma\left(\right.$ via $\left.\varphi_{b}\right)$ is larger than the second-order Schwarzschild term $\left(15 \pi M^{2} / 8 R^{2}\right)$ with the leading order term $M \varphi_{b}^{2} / R$ contributing positively to the Schwarzschild term $2 M / R$. This contribution is independent of $k$ and purely due to the other constant $\gamma$. However, note that this additional term is always positive, no matter what the sign of $\gamma$ is but the value is slightly more when $\gamma$ is taken with a positive sign, especially when the impact parameter $R \gg R_{0}$ inside the vacuole, which indicates that a positive sign $\gamma$ is preferable. The other contributions proportional to negative $k$ in (20) are much less and negligible for typical galactic values. The fact that the vacuole model provides a positive contribution to bending proportional to $\gamma$ is the main result of this paper.

\section{Conclusion}

The present paper has been inspired by the fact that, in recent times, MKdS solution considered by Weyl conformal gravity has seen remarkable successes in explaining the flat rotation curves and maximal sizes of galaxies without requiring the hypothesis of dark matter. However, with regard to light bending by the galaxies, when light has to traverse attractive dark matter, one would expect that light should bend more than the Schwarzschild value. This increase has actually been confirmed by experimental observations on gravitational lensing by galactic clusters [4]. The difficulty with the standard method is that it is not suitable for asymptotically nonflat spacetimes such as MKdS. Also, the original Rindler-Ishak method leads to imaginary values when applied to galactic scales. Thus, the improved method developed by them (using the concept of Einstein-Strauss vacuole) shows that the contribution due to $\gamma$ term is always positive thereby making the total first-order contribution in excess of the Schwarzschild value, as desired. The result of this paper places conformal gravity on a firmer footing with regard to astrophysical observations.

Finally, we should mention that the above result is supported also by a similar result in Cattani et al. [8] about the first-order bending being in excess of the Schwarzschild value. Their method is based on the original Rindler-Ishak method as developed in [5] appropriate to asymptotically nonflat situations. However, Sultana and Kazanas [9] used the standard method, which seems rather inappropriate in such situations. Moreover, their actual calculations are disfigured by certain errors that lead to a decrease from the Schwarzschild value, which is unphysical as the galactic halo is attractive.

\section{References}

[1] P. D. Mannheim and D. Kazanas, "Exact vacuum solution to conformal Weyl gravity and galactic rotation curves," Astrophysical Journal, vol. 342, p. 635, 1989.

[2] P. D. Mannheim and J. G. O'Brien, "Impact of a global quadratic potential on galactic rotation curves," Physical Review Letters, vol. 106, no. 12, Article ID 121101, 2011.

[3] K. K. Nandi and A. Bhadra, "Comment on 'Impact of a Global Quadratic Potential on Galactic Rotation Curves"' Physical Review Letters, vol. 109, no. 7, Article ID 079001, 2012.

[4] A. Edery and M. B. Paranjape, "Classical tests for Weyl gravity: deflection of light and time delay," Physical Review D, vol. 58, no. 2, Article ID 024011, 1998.

[5] W. Rindler and M. Ishak, "Contribution of the cosmological constant to the relativistic bending of light revisited," Physical Review D, vol. 76, no. 4, Article ID 043006, 2007.

[6] M. Ishak, W. Rindler, J. Dossett, J. Moldenhauer, and C. Allison, "A new independent limit on the cosmological constant/dark energy from the relativistic bending of light by Galaxies and clusters of Galaxies," Monthly Notices of the Royal Astronomical Society, vol. 388, no. 3, pp. 1279-1283, 2008.

[7] A. Einstein and E. Strauss, "The influence of the expansion of space on the gravitation fields surrounding the individual stars," Reviews of Modern Physics, vol. 17, no. 2-3, pp. 120-124, 1945. 
[8] C. Cattani, C. Cattani, M. Scalia, E. Laserra, I. Bochicchio, and K. K. Nandi, "Correct light deflection in Weyl conformal gravity," Physical Review D, vol. 87, no. 4, Article ID 047503, 2013.

[9] J. Sultana and D. Kazanas, "Bending of light in conformal Weyl gravity," Physical Review D, vol. 81, no. 12, Article ID 127502, 2010. 

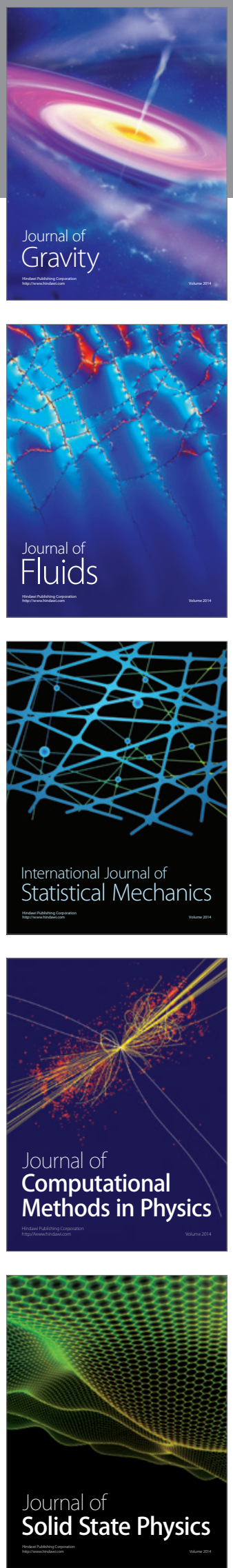

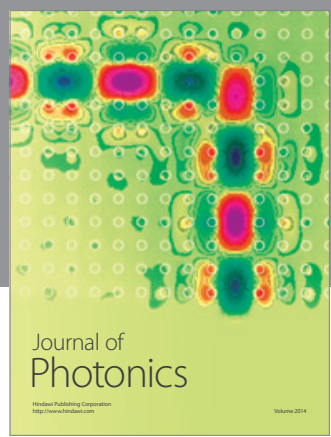

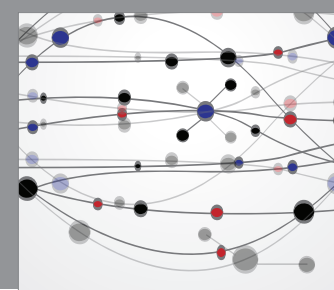

The Scientific World Journal

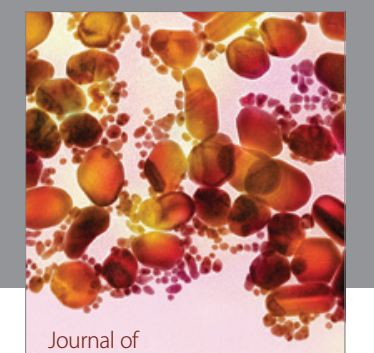

Soft Matter
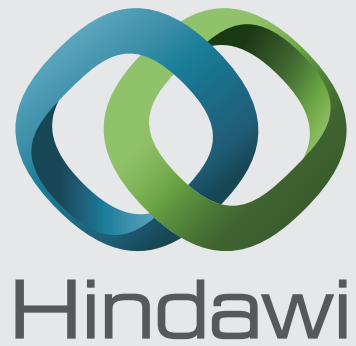

Submit your manuscripts at

http://www.hindawi.com
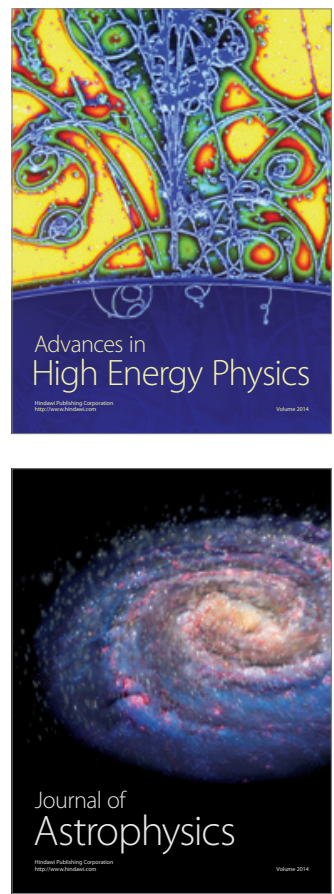
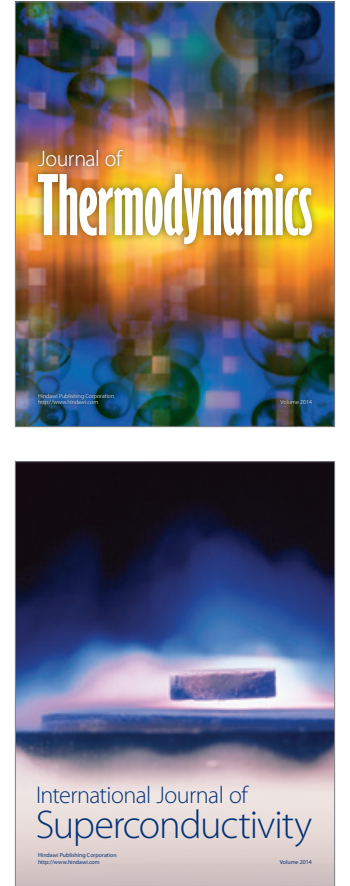
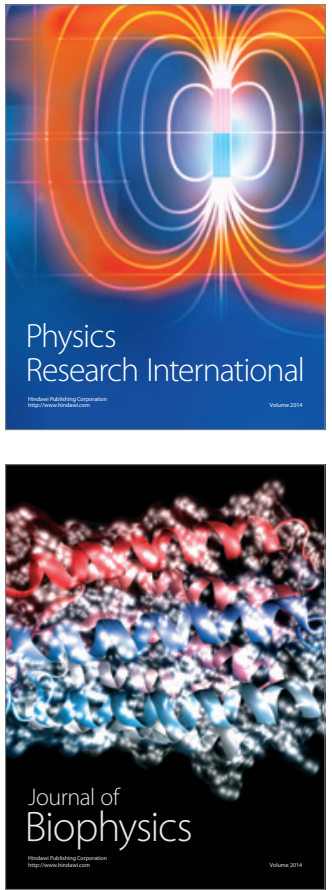
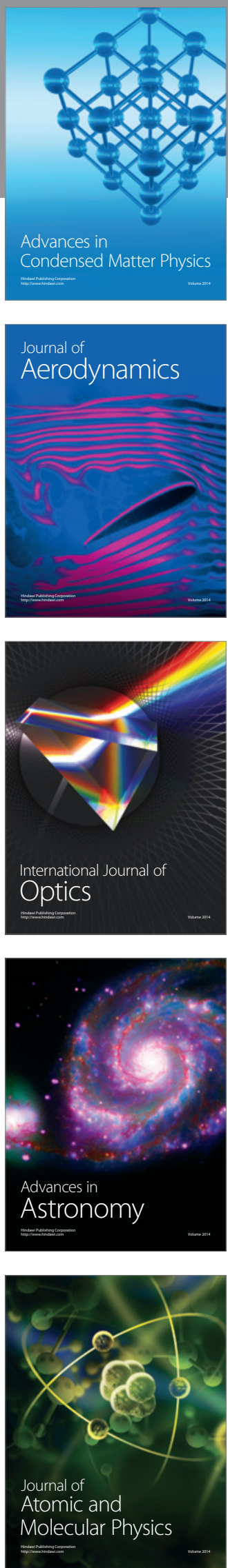